\title{
ON THE OPTIMALITY OF DOUBLE-BRACKET FLOWS
}

\section{ANTHONY M. BLOCH and ARIEH ISERLES}

\author{
Received 21 June 2004
}

\begin{abstract}
We analyze the optimality of the stable fixed point of the double-bracket equations. We introduce different types of optimality and prove local and global optimality results with respect to the Schatten $p$-norms.
\end{abstract}

2000 Mathematics Subject Classification: 15A42, 34D23.

1. Introduction. Double-bracket flows were introduced simultaneously by Brockett in the context of control theory and optimization [5] and by Chu and Driessel in connection with the computation of certain problems in numerical linear algebra [7]. Let $\operatorname{Sym}(n)$ denote the set of real, $n \times n$ symmetric matrices. A double-bracket flow (DBF) is the matrix differential equation

$$
\mathbf{Y}^{\prime}=[[\mathbf{N}, \mathbf{Y}], \mathbf{Y}], \quad t \geq 0, \quad \mathbf{Y}(0)=\mathbf{Y}_{0} \in \operatorname{Sym}(n),
$$

where $\mathbf{N} \in \operatorname{Sym}(n)$ and $[\cdot, \cdot]$ is the usual matrix commutator. Without loss of generality, we assume that $\mathbf{N}$ is a diagonal matrix, $\mathbf{N}=\operatorname{diag} \boldsymbol{\lambda}$. Otherwise, we may reduce (1.1) to this form: $\mathbf{Y}(t)=\mathbf{P} \tilde{\mathbf{Y}}(t) \mathbf{P}^{\top}$, where $\tilde{\mathbf{Y}}^{\prime}=[[\Lambda, \tilde{\mathbf{Y}}], \tilde{\mathbf{Y}}], \tilde{\mathbf{Y}}(0)=\mathbf{P}^{\top} \mathbf{Y}_{0} \mathbf{P}$, and $\mathbf{N}=\mathbf{P} \Lambda \mathbf{P}^{\top}$, with $\mathbf{P} \in \mathrm{SO}(n)$ and $\Lambda$ a diagonal matrix.

The Lie algebraic generalization of this flow was analyzed in [2, 3].

Equations of the form (1.1) share a number of features that render them of interest, both in the context of a number of applications and as mathematical objects in their own right.

Firstly, (1.1) is a special instance of an isospectral flow

$$
\mathbf{Y}^{\prime}=[\mathbf{B}(t, \mathbf{Y}), \mathbf{Y}], \quad t \geq 0, \quad \mathbf{Y}(0)=\mathbf{Y}_{0} \in \operatorname{Sym}(n),
$$

where $\mathbf{B}: \mathbb{R}_{+} \times \operatorname{Sym}(n) \rightarrow \mathfrak{s o}(n)$, the set $\mathfrak{s o}(n)$ being the Lie algebra of $n \times n$ real, skewsymmetric matrices. It easily follows that

$$
\mathbf{Y}(t)=\mathbf{Q}(t) Y_{0} \mathbf{Q}^{\top}(t), \quad t \geq 0,
$$

where

$$
\mathbf{Q}^{\prime}=\mathbf{B}\left(t, \mathbf{Q} \mathbf{Y}_{0} \mathbf{Q}^{\top}\right), \quad t \geq 0, \quad \mathbf{Q}(0)=\mathbf{I} .
$$

Since the latter is a Lie-group equation, we deduce that $\mathbf{Q}$ evolves in $\mathrm{SO}(n)$, therefore, by (1.3), $\mathbf{Y}$ is a similarity transformation of the initial value $\mathbf{Y}_{0}$ [15]. In other words, 
the eigenvalues of $\mathbf{Y}(t)$ in (1.2) (and, by implication, in the DBF (1.1)) are invariants of the flow and do not vary as $t$ increases. We note in passing that (1.3) is the key to practical computation of the solution of (1.2), whilst respecting its invariants [6, 14].

The second feature of the DBF (1.1) is that it is a gradient system, with a global Lyapunov function, therefore it is assured of convergence to a fixed point of the flow as $t \rightarrow \infty$ [5]. More precisely, as shown in [3, 4], it is a gradient flow with respect to the so-called normal metric on an appropriate adjoint orbit of a compact Lie group. This feature, of critical importance in applications, is in general invalid for arbitrary isospectral flows (1.2): thus, for example, the solution of

$$
\mathbf{Y}^{\prime}=[\mathbf{B}, \mathbf{Y}], \quad t \geq 0, \quad \mathbf{Y}(0)=\left[\begin{array}{ll}
a & b \\
b & c
\end{array}\right], \quad \text { where } \mathbf{B}=\left[\begin{array}{cc}
0 & 1 \\
-1 & 0
\end{array}\right]
$$

is

$$
\mathbf{Y}(t)=\left[\begin{array}{cc}
\frac{1}{2}(a+c)+b \sin 2 t+\frac{1}{2}(a-c) \cos 2 t & b \cos 2 t-\frac{1}{2}(a-c) \sin 2 t \\
b \cos 2 t-\frac{1}{2}(a-c) \sin 2 t & \frac{1}{2}(a+c)-b \sin 2 t-\frac{1}{2}(a-c) \cos 2 t
\end{array}\right],
$$

an oscillatory matrix.

It is generally true in fact that integrable Hamiltonian systems are described by isospectral flows. As shown by [1], however, in the case that the matrix $\mathbf{Y}$ in the DBF is tridiagonal and the matrix $\mathbf{N}$ is the diagonal matrix $\operatorname{diag}(1,2, \ldots, n)$, the DBF is both gradient and Hamiltonian on a level set of its integrals. The flow is the Toda lattice flow and the level set noncompact and diffeomorphic to a product of lines, unlike many Hamiltonian systems where the level set of the integrals is diffeomorphic to a torus. The seminal initial paper on the nonperiodic Toda flow is [21], where its gradient nature is demonstrated. Other early key papers include [8, 22, 23].

Since the existence of a global Lyapunov function is fundamental to the subject matter of this paper, we herewith reproduce a proof, following that of [5], as well as presenting a second, local Lyapunov function which is (to the best of our knowledge) new and helps to shed light on the asymptotic behavior of $\mathbf{Y}(t)$.

We remark that this global Lyapunov functional plays a key role in the work of [1] in proving the that the Toda flow is gradient and in the general analysis of double-bracket equations in [2]. The functional was used earlier in proving monotonicity properties for Toda flows in [9, 17]. For further related work, see the references in [2].

We define

$$
h(t)=\operatorname{tr}[\mathbf{Y}(t)-\mathbf{N}]^{2}=\operatorname{tr} \mathbf{Y}^{2}(t)+\operatorname{tr} \mathbf{N}^{2}-\operatorname{tr}[\mathbf{Y}(t) \mathbf{N}+\mathbf{N Y}(t)], \quad t \geq 0
$$

Both $\operatorname{tr} \mathbf{N}^{2}$ and $\operatorname{tr} \mathbf{Y}^{2}(t)$ are independent of $t$ : the first since $\mathbf{N}$ itself is a constant matrix and the second because thetrace is the sum of the eigenvalues and, since the eigenvalues 
of $\mathbf{Y}(t)$ remain fixed, so do also the eigenvalues of $\mathbf{Y}^{2}(t)$. We deduce that

$$
h^{\prime}(t)=-\frac{d}{d t} \operatorname{tr}[\mathbf{Y}(t) \mathbf{N}+\mathbf{N Y}(t)], \quad t \geq 0 .
$$

Since $\mathbf{N}=\operatorname{diag} \boldsymbol{\lambda}$, we have

$$
\begin{gathered}
(\mathbf{Y N}+\mathbf{N Y})_{k, l}=\left(\lambda_{k}+\lambda_{l}\right) Y_{k, l}, \quad k, l=1,2, \ldots, n, \\
\mathbf{Y}_{k, l}^{\prime}=[[\mathbf{N}, \mathbf{Y}], \mathbf{Y}]_{k, l}=\sum_{i=1}^{n}\left(\lambda_{k}-2 \lambda_{i}+\lambda_{l}\right) Y_{k, i} Y_{i, l}, \quad k, l=1,2, \ldots, n .
\end{gathered}
$$

Substituting the differential equation, (1.1), and the symmetry of $\mathbf{Y}$ yield

$$
\begin{aligned}
h^{\prime} & =-2 \sum_{k=1}^{n} \lambda_{k} Y_{k, k}^{\prime}=-4 \sum_{k=1}^{n} \lambda_{k} \sum_{i=1}^{n}\left(\lambda_{k}-\lambda_{i}\right) Y_{k, i}^{2} \\
& =-2 \sum_{k=1}^{n} \sum_{i=1}^{n} \lambda_{k}\left(\lambda_{k}-\lambda_{i}\right) Y_{k, i}^{2}-2 \sum_{i=1}^{n} \sum_{k=1}^{n} \lambda_{i}\left(\lambda_{i}-\lambda_{k}\right) Y_{i, k}^{2} \\
& =-2 \sum_{k=1}^{n} \sum_{i=1}^{n}\left(\lambda_{k}-\lambda_{i}\right)^{2} Y_{k, i}^{2} .
\end{aligned}
$$

Therefore, $h^{\prime}(t) \leq 0$ and, unless $\mathbf{Y}(t)$ is a diagonal matrix, $h^{\prime}(t)<0$. Since $(\mathbf{Y}-\mathbf{N})^{2}$ is positive semidefinite, the function $h$ is nonnegative and we deduce that $h$ is a global Lyapunov function and that $\lim _{t \rightarrow \infty} h(t)$ exists. This implies $\lim _{t \rightarrow \infty} h^{\prime}(t)=0$, therefore $\hat{\mathbf{Y}}=\lim _{t \rightarrow \infty} \mathbf{Y}(t)$ exists and, because of our analysis, is a diagonal matrix.

Needless to say, the diagonal elements of $\hat{\mathbf{Y}}$ are the eigenvalues of $\mathbf{Y}_{0}$ (hence of $\mathbf{Y}(t)$, $t \geq 0$ ), which we denote by $d_{1}, d_{2}, \ldots, d_{n}$. We henceforth assume for the sake of simplicity (and to avoid a welter of special cases of no great intrinsic significance) that the $2 n$ real numbers $\left\{d_{1}, d_{2}, \ldots, d_{n}\right\}$ and $\left\{\lambda_{1}, \lambda_{2}, \ldots, \lambda_{n}\right\}$ are distinct. Since any diagonal matrix annihilates the vector field in (1.1), all matrices of the from $\operatorname{diag} \mathbf{d}_{\boldsymbol{\pi}}$, where $\boldsymbol{\pi}$ is a permutation of $\{1,2, \ldots, n\}$, are fixed points of DBF. To enquire about their attractivity, we let $\mathbf{Y}(t)=\operatorname{diag} \mathbf{d}_{\boldsymbol{\pi}}+E(t)$, where $\|E(t)\|$ is small. Since diagonal matrices form a commutative algebra, we deduce that

$$
\begin{aligned}
E^{\prime} & =Y^{\prime}=\left[\left[N, \operatorname{diag} \mathbf{d}_{\boldsymbol{\pi}}+E\right], \operatorname{diag} \mathbf{d}_{\boldsymbol{\pi}}+E\right]=\left[[N, E], \operatorname{diag} \mathbf{d}_{\boldsymbol{\pi}}+E\right] \\
& =\left[[N, E], \operatorname{diag} \mathbf{d}_{\boldsymbol{\pi}}\right]+\mathcal{O}\left(\|E\|^{2}\right) .
\end{aligned}
$$

Therefore, for every $k, l=1,2, \ldots, n$,

$$
\begin{aligned}
E_{k, l}^{\prime} & =\left(\lambda_{k} d_{\pi_{l}}-\lambda_{l} d_{\pi_{l}}-\lambda_{k} d_{\pi_{k}}+\lambda_{l} d_{\pi_{k}}\right) E_{k, l}+\mathcal{O}\left(\|E\|^{2}\right) \\
& =-\left(\lambda_{k}-\lambda_{l}\right)\left(d_{\pi_{k}}-d_{\pi_{l}}\right) E_{k, l}+\mathcal{O}\left(\|E\|^{2}\right) .
\end{aligned}
$$

We deduce that each $E_{k, l}$ decays (at an exponential speed) if and only if

$$
\left(\lambda_{k}-\lambda_{l}\right)\left(d_{\pi_{k}}-d_{\pi_{l}}\right)>0, \quad k, l=1,2, \ldots, n, k \neq l .
$$

Therefore, there is just one attractive fixed point $\hat{\mathbf{Y}}$, given by the permutation of $\mathbf{d}$ that arranges the eigenvalues in the same order as the $\boldsymbol{\lambda} s$. 
Next, we construct a local Lyapunov function,

$$
m(t)=\sum_{k=1}^{n} \sum_{\substack{l=1 \\ l \neq k}}^{n} Y_{k, l}^{2}(t)=\sum_{k=1}^{n} \sum_{l=1}^{n} Y_{k, l}^{2}(t)-\sum_{k=1}^{n} Y_{k, k}^{2}(t)=\|\mathbf{Y}(t)\|_{F}^{2}-\sum_{k=1}^{n} Y_{k, k}^{2}(t)
$$

where $\|\cdot\|_{F}$ is the Frobenius norm. Since $\|\mathbf{A}\|_{F}^{2}$ equals the sum of squares of the eigenvalues of a real, square matrix $\mathbf{A}$, we use isospectrality to argue that $\|\mathbf{Y}(t)\|_{F}^{2}$ is constant. Therefore, we deduce from (1.9) that

$$
\begin{aligned}
m^{\prime}(t) & =-2 \sum_{k=1}^{n} Y_{k, k} Y_{k, k}^{\prime}=-4 \sum_{k=1}^{n} Y_{k, k} \sum_{i=1}^{n}\left(\lambda_{k}-\lambda_{i}\right) Y_{k, i}^{2} \\
& =-2 \sum_{k=1}^{n} \sum_{i=1}^{n}\left(\lambda_{k}-\lambda_{i}\right) Y_{k, k} Y_{k, i}^{2}-2 \sum_{i=1}^{n} \sum_{k=1}^{n}\left(\lambda_{i}-\lambda_{k}\right) Y_{i, i} Y_{k, i}^{2} \\
& =-2 \sum_{k=1}^{n} \sum_{i=1}^{n}\left(\lambda_{k}-\lambda_{i}\right)\left(Y_{k, k}-Y_{i, i}\right) Y_{k, i}^{2} .
\end{aligned}
$$

We have already seen that, unless $\mathbf{Y}_{0}$ is a diagonal matrix, the solution $\mathbf{Y}$ tends to the diagonal matrix consistently with (1.13). Therefore, there exists $t^{*} \geq 0$ such that for every $t \geq t^{*}$ the vector $\operatorname{diag} \mathbf{Y}(t)$ lies in the closed, nonempty set

$$
\left\{\mathbf{x} \in \mathbb{R}^{n}:\left(\lambda_{k}-\lambda_{i}\right)\left(x_{k}-x_{i}\right) \geq 0, k, l=1,2 \ldots, n\right\}
$$

As we have just demonstrated, within this neighborhood of the attractive fixed point, $m \geq 0$ is a local Lyapunov function and the Euclidean norm of off-diagonal elements is decaying monotonically.

The third important feature of the DBF (1.1) is that its fixed point $\hat{\mathbf{Y}}$, a diagonal matrix with $\mathbf{d}_{\boldsymbol{\pi}}$ along the diagonal (cf. (1.13)) optimizes the distance to $\mathbf{N}$ in the Frobenius norm.

As a matter of fact, one can distinguish between the following three types of optimality.

(1) Discrete optimality. The Frobenius distance from $\mathbf{N}$ is minimized at $\hat{\mathbf{Y}}$ among all the $n$ ! diagonal matrices similar to $\mathbf{Y}_{0}$. This relatively weak form of optimality is the one considered (and proved) by [5]. Indeed, in Brockett's terminology, the DBF (1.1) is an "analogue computer" for the calculation of eigenvalues, sorting of lists and the solution of linear programming problems, and this makes discrete optimality a natural concept.

(2) Isospectral optimality. Given an arbitrary matrix norm $\|\cdot\|$, is it true that (without loss of generality) for all diagonal matrices $\mathbf{N}$ and all $\mathbf{Y}_{0} \in \operatorname{Sym}(n)$ the inequality $\| \hat{\mathbf{Y}}$ $\mathbf{N}\|\leq\| \mathbf{X}-\mathbf{N} \|$ holds for all $\mathbf{X} \in \operatorname{Sym}(n)$ similar to $\mathbf{Y}_{0}$, such that $\|\mathbf{X}-\hat{\mathbf{Y}}\|$ is sufficiently small? In this case, we say that the norm is isospectrally optimal. Moreover, if the inequality is (for every $\mathbf{Y}_{0}$ and $\mathbf{N}$ ) sharp for $\mathbf{X} \neq \hat{\mathbf{Y}}$ in an arbitrarily small neighbourhood, except perhaps for a lower-dimensional submanifold, we say that the norm optimality is strong. If for some $\mathbf{Y}_{0}$ and $\mathbf{N}$ we have equality in a proper neighbourhood of $\hat{\mathbf{Y}}$ then optimality is weak. 
(3) Global optimality. This is the strongest possible form of optimality,

$$
\|\hat{\mathbf{Y}}-\mathbf{N}\| \leq\|\mathbf{X}-\mathbf{N}\|, \quad \mathbf{X} \in \operatorname{Sym}(n), \mathbf{X} \text { similar to } \mathbf{Y}_{0} .
$$

Intriguingly, although never mentioned in [5], it is implicit in the analysis of that paper that the Frobenius norm is globally optimal. This follows at once from $h^{\prime}(t) \leq 0$ (since $h(t)=\|Y(t)-\mathbf{N}\|_{F}^{2}$ ), the fact that $\hat{Y}$ is the only fixed point for nondiagonal $\mathbf{Y}_{0} \mathrm{~s}$ and discrete optimality. Given that all these facts are already present in [5], it is only fair to attribute the global optimality of the Frobenius norm to Brockett.

In this paper, we wish to focus on isospectral optimality for different unitarily invariant norms: a matrix norm $\|\cdot\|$ is unitarily invariant if $\|\mathbf{P X Q}\|=\|\mathbf{X}\|$ for every $\mathbf{P}, \mathbf{Q} \in \mathrm{SO}(n), \mathbf{X} \in \mathrm{M}_{n, n}$ [13, page 203]. We commence Section 2 with von Neumann's celebrated characterization of unitarily invariant norms in terms of symmetric gauges $[24,25]$. The most important family of unitarily invariant norms are the so-called Schatten $p$-norms, $1 \leq p \leq \infty$. In the rest of the section, we determine the range of $p$ such that a Schatten $p$-norm is isospectrally optimal.

We remark that the derivative of the Frobenius norm $h(t)=\|\mathbf{Y}(t)-\mathbf{N}\|_{F}^{2}$ along the flow of the double-bracket equations reduces to the derivative of $\operatorname{tr} \mathbf{Y N}$ which is precisely the gradient function for the flow. In this case, global optimality follows as in paragraph (3) above. The key difficulty in the current paper is that the more general norms we discuss here involve higher powers of $\mathbf{Y}$.

In Section 3, we discuss the case $n=2$. Although the solution of (1.1) can be easily written down in an explicit fashion, isospectral optimality is far from being trivial. We resolve this issue completely and characterize all isospectrally optimal norms for $n=2$. We also show that all Schatten $p$-norms are globally optimal for $n=2$.

2. Schatten $p$-norms. A vector norm $|\cdot|: \mathbb{R}^{n} \rightarrow \mathbb{R}_{+}$is said to be symmetric if $|\mathbf{P x}|=$ $|\mathbf{x}|$ for every $\mathbf{x} \in \mathbb{R}^{n}$ and every permutation matrix $\mathbf{P} \in \mathrm{M}_{n, n}$, and absolute if $|\mathbf{x}|=\|\mathbf{x}\|$, $\mathbf{x} \in \mathbb{R}^{n}$, where $|\mathbf{x}|$ is the vector of the absolute values of the elements of $\mathbf{x}$. If $|\cdot|$ is both symmetric and absolute, it is called a symmetric gauge $[13,18]$. Thus, for example, every $\ell_{p}\left[\mathbb{R}^{n}\right]$ norm is a symmetric gauge, but no nontrivial weighted $\ell_{p}\left[\mathbb{R}^{n}\right]$ norm is symmetric and the norm $\left|\left[x_{1}, x_{2}\right]^{\top}\right|=\left[x_{1}^{2}+x_{2}^{2}+\left(x_{1}-x_{2}\right)^{2}\right]^{1 / 2}$ is not positive. (We leave it as an elementary exercise to the reader to prove that this is indeed a norm. perhaps the easiest proof follows by showing that its unit ball is convex.)

Given $\mathbf{X} \in \mathrm{M}_{n, n}$, we denote by $\operatorname{sing}(\mathbf{X})$ the vector of singular values of $\mathbf{X}$ : to ensure proper definition, we assume that the elements of $\operatorname{sing}(\mathbf{X})$ are arranged in increasing order, but this will play no further role in our discussion. Note that for $\mathbf{X} \in \operatorname{Sym}(n)$ the map $\operatorname{sing}(\mathbf{X})$ produces the absolute values of the eigenvalues of the matrix.

THEOREM 2.1 [24, 25]. A matrix norm $\|\cdot\|$ is unitarily invariant if and only if there exists a symmetric gauge $|\cdot|$ such that

$$
\|\mathbf{X}\|=|\operatorname{sing}(\mathbf{X})|, \quad \mathbf{X} \in \mathrm{M}_{n, n} .
$$

We have already mentioned that the standard $\ell_{p}\left[\mathbb{R}^{n}\right]$ norm, $1 \leq p \leq \infty$, is a symmetric gauge. Therefore, by Theorem 2.1, it gives rise to a unitarily-invariant norm, 
the Schatten $p$-norm $\|\cdot\|_{p}=|\operatorname{sing}(\cdot)|_{p}[13$, page 199]. In particular, the special choices $p \in\{1,2, \infty\}$ are important enough to deserve special names:

$\|\mathbf{X}\|_{1}=\mathbf{1}^{\top} \operatorname{sing}(\mathbf{X})$ : the Ky Fan norm [13, page 195],

$\|\mathbf{X}\|_{2}=\|\mathbf{X}\|_{F}$ : the Frobenius norm,

$\|\mathbf{X}\|_{\infty}=\max \left(|\mathbf{X v}|_{2} /|\mathbf{v}|_{2}\right)$ : the (operator) Euclidean norm.

Noting in passing that the Schatten 2-norm is isospectrally optimal, we seek to characterize the range of $p \in[1, \infty]$ for which a Schatten $p$-norm shares this feature.

Assuming that $\mathbf{Y}_{0}$ is not already a diagonal matrix, we have proved in Section 1 that the flow (1.1) converges to $\hat{\mathbf{Y}}=\operatorname{diag} \mathbf{d}_{\boldsymbol{\pi}}$, where the permutation $\boldsymbol{\pi}$ is consistent with (1.13). We consider matrices $\mathbf{Y}_{\varepsilon} \in \operatorname{Sym}(n)$ from an isospectral orbit of $\hat{\mathbf{Y}}$ (i.e., similar to $\hat{\mathbf{Y}})$ within distance $0<\varepsilon \ll 1$ from $\hat{\mathbf{Y}}$. By construction, there exists a nonzero $\Omega \in \mathfrak{s o}(n)$ such that

$$
\mathbf{Y}_{\varepsilon}=\mathrm{e}^{\varepsilon \Omega} \hat{\mathbf{Y}} \mathrm{e}^{-\varepsilon \Omega}=\mathrm{e}^{\varepsilon \operatorname{ad}_{\Omega}} \hat{\mathbf{Y}}=\hat{\mathbf{Y}}+\varepsilon[\Omega, \hat{Y}]+\frac{1}{2} \varepsilon^{2}[\Omega,[\Omega, \hat{\mathbf{Y}}]]+\mathcal{O}\left(\varepsilon^{3}\right),
$$

where $\operatorname{ad}_{\mathbf{A}} \mathbf{B}=[\mathbf{A}, \mathbf{B}]$ is the adjoint operator of the Lie algebra $\mathfrak{s o}(n)$.

Given $\mathbf{A}, \mathbf{B} \in \operatorname{Sym}(n)$ and small $\delta>0$, we denote the eigenvalues of $\mathbf{A}+\delta \mathbf{B}$ by $\theta_{1}(\delta)$, $\theta_{2}(\delta), \ldots, \theta_{n}(\delta)$. Provided that $\left\{\theta_{1}(0), \theta_{2}(0), \ldots, \theta_{n}(0)\right\}$, the spectrum of $\mathbf{A}$ is distinct, a classical perturbation result is

$$
\theta_{k}(\delta)=\theta_{k}(0)+\delta \mathbf{v}_{k}^{\top} \mathbf{B} \mathbf{v}_{k}+\delta^{2} \sum_{\substack{i=1 \\ i \neq k}}^{n} \frac{\left(\mathbf{v}_{k}^{\top} \mathbf{B} \mathbf{v}_{i}\right)^{2}}{\theta_{k}(0)-\theta_{i}(0)}+\mathcal{O}\left(\delta^{3}\right)
$$

where $\mathbf{v}_{k}$ is an eigenvector of $\mathbf{A}$ corresponding to the eigenvalue $\theta_{k}(0)$, such that $\left\|\mathbf{v}_{k}\right\|_{2}=1$ [28, page 70]. We now let

$$
\mathbf{A}=\hat{\mathbf{Y}}-\mathbf{N}, \quad \mathbf{B}=\varepsilon^{-1}\left(\mathbf{Y}_{\varepsilon}-\hat{\mathbf{Y}}\right)=[\Omega, \hat{Y}]+\frac{1}{2} \varepsilon[\Omega,[\Omega, \hat{\mathbf{Y}}]]+\mathcal{O}\left(\varepsilon^{2}\right)
$$

and $\delta=\varepsilon$. Since the eigenvalues of the diagonal matrix $\hat{\mathbf{Y}}-\mathbf{N}$ are $\eta_{k}:=d_{\pi_{k}}-\lambda_{k}, k=$ $1,2, \ldots, n$ and the corresponding normalized eigenvectors are the unit vectors $\mathbf{e}_{k},(2.3)$ yields

$$
\theta_{k}(\varepsilon)=\eta_{k}+\varepsilon \mathbf{e}_{k}^{\top}[\Omega, \hat{\mathbf{Y}}] \mathbf{e}_{k}+\varepsilon^{2}\left\{\frac{1}{2} \mathbf{e}_{k}^{\top}[\Omega,[\Omega, \hat{Y}]] \mathbf{e}_{k}+\sum_{i \neq k} \frac{\left(d_{\pi_{k}}-d_{i}\right)^{2}}{\eta_{k}-\eta_{i}} \Omega_{k, i}^{2}\right\}+\mathcal{O}\left(\varepsilon^{3}\right)
$$

However, because of the skew symmetry of $[\Omega, \hat{\mathbf{Y}}]$ and diagonality of $\hat{\mathbf{Y}}$, it is true that

$$
\begin{gathered}
\mathbf{e}_{k}^{\top}[\Omega, \hat{\mathbf{Y}}] \mathbf{e}_{k}=0, \\
\mathbf{e}_{k}^{\top}[\Omega,[\Omega, \hat{\mathbf{Y}}]] \mathbf{e}_{k}=-\sum_{i=1}^{n}\left(d_{\pi_{k}}-d_{\pi_{i}}\right) \Omega_{k, i}^{2}
\end{gathered}
$$


Therefore, the $O(\varepsilon)$ term vanishes and

$$
\begin{aligned}
\theta_{k}(\varepsilon) & =\eta_{k}+\varepsilon^{2} \sum_{i \neq k} \frac{d_{\pi_{k}}-d_{\pi_{i}}}{\eta_{k}-\eta_{i}}\left(d_{\pi_{k}}-d_{\pi_{i}}-\eta_{k}+\eta_{i}\right) \Omega_{k, i}^{2}+\mathcal{O}\left(\varepsilon^{3}\right) \\
& =\eta_{k}+\varepsilon^{2} \sum_{i \neq k} \frac{\left(d_{\pi_{k}}-d_{\pi_{i}}\right)\left(\lambda_{k}-\lambda_{i}\right)}{\eta_{k}-\eta_{i}} \Omega_{k, i}^{2}+\mathcal{O}\left(\varepsilon^{3}\right) \\
& =\eta_{k}+\varepsilon^{2} \mu_{k}+\mathcal{O}\left(\varepsilon^{3}\right),
\end{aligned}
$$

where

$$
\mu_{k}=\sum_{i \neq k} \frac{\left(d_{\pi_{k}}-d_{\pi_{i}}\right)\left(\lambda_{k}-\lambda_{i}\right)}{d_{\pi_{k}}-d_{\pi_{i}}-\lambda_{k}+\lambda_{i}} \Omega_{k, i}^{2}
$$

Letting $\|\cdot\|=|\operatorname{sing}(\cdot)|$, a unitarily invariant norm, and assuming that $|\cdot|$ is (locally) differentiable, we denote by $\mathbf{g}$ the gradient of the symmetric gauge,

$$
g_{k}(\mathbf{x})=\frac{\partial|\mathbf{x}|}{\partial x_{k}}, \quad k=1,2, \ldots, n
$$

Since $\mathbf{Y}_{\varepsilon}-\mathbf{N} \in \operatorname{Sym}(n)$, its singular values are $\left|\theta_{k}(\varepsilon)\right|, k=1,2, \ldots, n$. Therefore,

$$
\left\|\mathbf{Y}_{\varepsilon}-\mathbf{N}\right\|=|\boldsymbol{\theta}(\varepsilon)|=\left|\boldsymbol{\theta}(0)+\varepsilon^{2} \boldsymbol{\mu}+\mathbb{O}\left(\varepsilon^{3}\right)\right|=\|\hat{\mathbf{Y}}-\mathbf{N}\|+\varepsilon^{2} \boldsymbol{\mu}^{\top} \mathbf{g}(\boldsymbol{\eta})+\mathbb{O}\left(\varepsilon^{3}\right) .
$$

We deduce that the DBF (1.1) is isospectrally optimal if and only if

$$
\boldsymbol{\mu}^{\top} \mathbf{g}(\boldsymbol{\eta}) \geq 0
$$

and the inequality is sharp for at least one $\Omega \in \mathfrak{s o}(n)$.

We specialize our argument to Schatten $p$-norms, restricting $p$ at the first instance to a finite value, $1 \leq p<\infty$. In that case, it is easy to calculate the gradient

$$
g_{k}(\mathbf{x})=\frac{\left|x_{k}\right|^{p-1}}{|\mathbf{x}|_{p}^{p-1}} \operatorname{sgn} x_{k}, \quad k=1,2, \ldots, n,
$$

with derivative singularities when a component of $\mathbf{x}$ is zero. (These derivative discontinuities do not matter since we apply the above formula in the sequel exclusively to vectors with nonvanishing components.) Let

$$
U_{k, i}=\left(d_{\pi_{k}}-d_{\pi_{i}}\right)\left(\lambda_{k}-\lambda_{i}\right) \Omega_{k, i}^{2}, \quad k, i=1,2, \ldots, n
$$

Since $\hat{\mathbf{Y}}$ is the attractive fixed point, it follows from (1.13) that $U_{k, i} \geq 0$ for all $k, i=$ $1,2, \ldots, n$ and, since $\Omega$ is nonzero, so is the symmetric matrix $\mathbf{U}$. The numbers $\eta_{k}$ are all nonzero, since $\mathbf{d}$ and $\boldsymbol{\lambda}$ are all distinct, therefore we may partition the set $\{1,2, \ldots, n\}$ into the union $\mathscr{I}_{-} \cup \mathscr{I}_{+}$, where

$$
\eta_{k}<0 \Longrightarrow k \in \mathscr{I}_{-}, \quad \eta_{k}>0 \Longrightarrow k \in \mathscr{I}_{+} .
$$


For the Schatten $p$-norm we thus have

$$
\begin{aligned}
|\boldsymbol{\eta}|_{p}^{p-1} \boldsymbol{\mu}^{\top} \mathbf{g}(\boldsymbol{\eta})= & \sum_{k=1}^{n} \sum_{\substack{l=1 \\
l \neq k}}^{n} \frac{U_{k, l}}{\eta_{k}-\eta_{l}}\left|\eta_{k}\right|^{p-1} \operatorname{sgn} \eta_{k} \\
= & \sum_{k \in \mathscr{I}_{-}} \sum_{l \in \mathscr{I}_{-} \backslash\{k\}} \frac{\left|\eta_{k}\right|^{p-1} U_{k, l}}{\left|\eta_{k}\right|-\left|\eta_{l}\right|}+\sum_{k \in \mathscr{I}_{+}} \sum_{l \in \mathscr{I}_{+} \backslash\{k\}} \frac{\left|\eta_{k}\right|^{p-1} U_{k, l}}{\left|\eta_{k}\right|-\left|\eta_{l}\right|} \\
& +\sum_{k \in \mathscr{I}_{-}} \sum_{l \in \mathscr{I}_{+}} \frac{\left|\eta_{k}\right|^{p-1} U_{k, l}}{\left|\eta_{k}\right|+\left|\eta_{l}\right|}+\sum_{k \in \mathscr{I}_{+}} \sum_{l \in \mathscr{I}_{-}} \frac{\left|\eta_{k}\right|^{p-1} U_{k, l}}{\left|\eta_{k}\right|+\left|\eta_{l}\right|} .
\end{aligned}
$$

The last two sums are nonnegative. Moreover, $\mathbf{U}$ being symmetric, with nonnegative entries,

$$
\begin{aligned}
& \sum_{k \in \mathscr{I}_{ \pm}} \sum_{l \in \mathscr{I}_{ \pm} \backslash\{k\}} \frac{\left|\eta_{k}\right|^{p-1} U_{k, l}}{\left|\eta_{k}\right|-\left|\eta_{l}\right|} \\
& \quad=\frac{1}{2} \sum_{k \in \mathscr{I}_{ \pm}} \sum_{l \in \mathscr{I}_{ \pm} \backslash\{k\}} \frac{\left|\eta_{k}\right|^{p-1} U_{k, l}}{\left|\eta_{k}\right|-\left|\eta_{l}\right|}+\frac{1}{2} \sum_{l \in \mathscr{I}_{ \pm}} \sum_{k \in \mathscr{I}_{ \pm} \backslash\{l\}} \frac{\left|\eta_{l}\right|^{p-1} U_{k, l}}{\left|\eta_{l}\right|-\left|\eta_{k}\right|} \\
& \quad=\sum_{l \in \mathscr{I}_{ \pm}} \sum_{k \in \mathscr{I}_{ \pm} \backslash\{l\}} \frac{\left|\eta_{k}\right|^{p-1}-\left|\eta_{l}\right|^{p-1}}{\left|\eta_{k}\right|-\left|\eta_{l}\right|} U_{k, l} \geq 0 .
\end{aligned}
$$

We conclude that $\boldsymbol{\mu}^{\top} \mathbf{g}(\boldsymbol{\eta}) \geq 0$. Moreover, examining the expressions above, we observe that, unless $p=1$ and the first two sums in (2.15) are identically zero, necessarily $\boldsymbol{\mu}^{\top} \mathbf{g}(\boldsymbol{\eta})>0$, because $\mathbf{U} \neq \mathbf{O}$. Therefore, because of (2.11), the Schatten $p$-norm is strongly isospectrally optimal for $p \in(1, \infty)$.

For the Ky Fan norm, $p=1$, the inner product $\boldsymbol{\mu}^{\top} \mathbf{g}(\boldsymbol{\eta})$ is zero in the special case of all the $\eta_{k}$ s being of the same sign. This, of course, need not necessarily imply that strong isospectral optimality fails, since, in principle, it might be assured by higherorder terms. Moreover, it is true that $\|\mathbf{Y}(t)-\mathbf{N}\|_{1} \geq\|\hat{\mathbf{Y}}-\mathbf{N}\|_{1}$ by continuity: since for every nonsingular A,

$$
\|\mathbf{A}\|_{1+\varepsilon}=\|A\|_{1}+\varepsilon\left[\sum_{k=1}^{n}\left|\sigma_{k}(\mathbf{A})\right| \log \left|\sigma_{k}(\mathbf{A})\right|-\log \sum_{k=1}^{n}\left|\sigma_{k}(\mathbf{A})\right|\right]+\mathcal{O}\left(\varepsilon^{2}\right),
$$

where $\sigma_{k}(\mathbf{A})$ are the singular values of $\mathbf{A}$, this follows from isospectral optimality for $p>1$. However, it is perfectly possible that the norm is "flat" in a nonempty open neighborhood of $\hat{\mathbf{Y}}$, implying weak optimality. Thus, note that $\operatorname{tr}[\mathbf{Y}(t)-\mathbf{N}]=\mathbf{1}^{\top}(\mathbf{d}-$ $\boldsymbol{\lambda}$ ) is independent of $t$. As long as all the entries along the diagonal of $\mathbf{Y}(t)-\mathbf{N}$ are nonnegative, it is true that

$$
\|\mathbf{Y}(t)-\mathbf{N}\|_{1}=\operatorname{tr}[\mathbf{Y}(t)-\mathbf{N}] \equiv \mathbf{1}^{\top}(\mathbf{d}-\boldsymbol{\lambda}) .
$$

Therefore, there always exists $t^{*} \geq 0$ such that $\|\mathbf{Y}(t)-\mathbf{N}\|_{1}$ is constant for all $t \geq t^{*}$ and there is no isospectral optimality. If (nonpermuted) $\mathbf{d}$ is consistent with (1.13), then it is perfectly possible that we may set $t^{*}=0$ and the Ky Fan norm of $\mathbf{Y}(t)-\mathbf{N}$ is always flat. This is the case if $n=2$ and, for example, $\min d_{k}>\max \lambda_{k}$ (cf. Section 3). 
Finally, we consider the Schatten $\infty$-norm (known also as the operator Euclidean norm). Clearly, it must be at the very least weakly isospectrally optimal, because of a continuity argument similar to the one we have used for the Ky Fan norm. Since, by our analysis,

$$
\left\|\mathbf{Y}_{\varepsilon}-\mathbf{N}\right\|_{p}^{p}=\|\hat{\mathbf{Y}}-\mathbf{N}\|_{p}^{p}+\varepsilon^{2} p \sum_{k=1}^{n}\left(\operatorname{sgn} \eta_{k}\right)\left|\eta_{k}\right|^{p-1} \sum_{i \neq k} \frac{U_{k, i}}{\eta_{k}-\eta_{i}}+\mathcal{O}\left(\varepsilon^{3}\right),
$$

taking the $p$ th root and letting $p \rightarrow \infty$ results in

$$
\left\|\mathbf{Y}_{\varepsilon}-\mathbf{N}\right\|_{\infty}=\|\hat{\mathbf{Y}}-\mathbf{N}\|_{\infty}+\varepsilon^{2} \operatorname{sgn} \eta_{r} \sum_{\substack{k=1 \\ k \neq r}}^{n} \frac{U_{r, k}}{\eta_{r}-\eta_{k}},
$$

where $\left|\eta_{r}\right|=\max _{i=1,2, \ldots, n}\left|\eta_{i}\right|$. We assume without loss of generality that $\eta_{r}>0$ (the proof for $\eta_{r}<0$ is identical). Then, $\eta_{r}-\eta_{k}>0, k=1,2, \ldots, n$, and since $U_{r, k} \geq 0$, $r, k=1,2, \ldots, n$, we deduce that

$$
\left\|\mathbf{Y}_{\varepsilon}-\mathbf{N}\right\|_{\infty} \geq\|\hat{\mathbf{Y}}-\mathbf{N}\|_{\infty}
$$

with an equality only if $\Omega_{r, k}=\Omega_{k, r}=0, k=1,2, \ldots, n$. Therefore, except for a lowerdimensional submanifold, the inequality is strict and the norm is strongly isospectrally optimal.

To sum up the results of this section, we have proved the following result.

THEOREM 2.2. The Schatten $p$-norm is strongly isospectrally optimal for $1<p \leq \infty$ and weakly isospectrally optimal for $p=1$.

3. Isospectral optimality for $n=2$. Given (1.1) with $n=2$, we have just three variables and, because the eigenvalues of $\mathbf{Y}(t)$ are invariant, possess just one degree of freedom. No wonder, thus, that the solution can be easily represented explicitly. Further, the equations in this case are just equivalent to the Toda lattice equations since a two-by-two matrix is of course tridiagonal. Letting

$$
\mathbf{N}=\left[\begin{array}{cc}
\lambda_{1} & 0 \\
0 & \lambda_{2}
\end{array}\right], \quad \mathbf{Y}_{0}=\left[\begin{array}{ll}
\alpha & \beta \\
\beta & \gamma
\end{array}\right]
$$

we have $d_{1}=(1 / 2)(\alpha+\gamma-\sigma), d_{2}=(1 / 2)(\alpha+\gamma+\sigma)$, where $\sigma=\sqrt{(\alpha-\gamma)^{2}+4 \beta^{2}}$. Moreover, as can be easily verified by direct differentiation, the solution of (1.1) is

$$
\begin{aligned}
Y_{1,1}(t) & =\frac{1}{2}(\alpha+\gamma)+\frac{1}{2} \sigma \tanh \left(\sigma \delta t+\frac{1}{2} \log \frac{\sigma+\alpha-\gamma}{\sigma-\alpha+\gamma}\right), \\
Y_{1,2}(y) & =Y_{2,1}(t)=\frac{\sigma \operatorname{sgn} \beta}{2 \cosh (\sigma \delta t+(1 / 2) \log ((\sigma+\alpha-\gamma) /(\sigma-\alpha+\gamma)))}, \\
Y_{2,2} & =\frac{1}{2}(\alpha+\gamma)-\frac{1}{2} \sigma \tanh \left(\sigma \delta t+\frac{1}{2} \log \frac{\sigma+\alpha-\gamma}{\sigma-\alpha+\gamma}\right),
\end{aligned}
$$

where $\delta=\lambda_{1}-\lambda_{2}$. 
Alternatively, we may express $\mathbf{Y}$ in terms of $d_{1}$ and $d_{2}$. A general matrix on the isospectral orbit is necessarily of the form

$$
\mathbf{Y}=\left[\begin{array}{cc}
\frac{1}{2}\left(d_{1}+d_{2}\right)-f & \pm \sqrt{\frac{1}{2}\left(d_{1}-d_{2}\right)^{2}-f^{2}} \\
\pm \sqrt{\frac{1}{2}\left(d_{1}-d_{2}\right)^{2}-f^{2}} & \frac{1}{2}\left(d_{1}+d_{2}\right)+f
\end{array}\right],
$$

where $|f| \leq(\sqrt{2} / 2)\left|d_{1}-d_{2}\right|$. If $\mathbf{Y}$ is a function of $t$, then so is $f$ and substitution in (1.1) yields, regardless of the sign of $Y_{1,2}=Y_{2,1}$, the Riccati equation

$$
f^{\prime}=2\left(\lambda_{2}-\lambda_{1}\right)\left[\frac{1}{4}\left(d_{2}-d_{1}\right)^{2}-f^{2}\right], \quad t \geq 0, \quad f(0)=f_{0},
$$

where $f_{0}$ is given by $\mathbf{Y}_{0}$. Its solution is

$$
f(t)=\frac{1}{2}\left|d_{1}-d_{2}\right| \frac{\tilde{f}+\tanh \left[\sqrt{\left|\lambda_{2}-\lambda_{1}\right| \cdot\left|d_{2}-d_{1}\right|} t\right]}{1+\tilde{f} \tanh \left[\sqrt{\left|\lambda_{2}-\lambda_{1}\right| \cdot\left|d_{2}-d_{1}\right|} t\right]}, \quad t \geq 0,
$$

where $\tilde{f}=2 f_{0} /\left|d_{1}-d_{2}\right|$.

We next explore global optimality for $n=2$. We commence by noting from (3.3) that the eigenvalues of $\mathbf{Y}(t)-\mathbf{N}$ are

$$
\kappa_{ \pm}(t)=\frac{1}{2}\left(d_{1}+d_{2}-\lambda_{1}-\lambda_{2}\right) \pm \sqrt{\left(d_{2}-d_{1}\right)^{2}+\left(\lambda_{2}-\lambda_{1}\right)^{2}-4\left(\lambda_{2}-\lambda_{1}\right) f(t)} .
$$

THEOREM 3.1. In the case $n=2$, all unitarily invariant norms are globally optimal.

Proof. Let $q=\left(d_{2}-d_{1}\right)^{2}+\left(\lambda_{2}-\lambda_{1}\right)^{2}, r=d_{1}+d_{2}-\lambda_{1}-\lambda_{2}$, therefore,

$$
\kappa_{ \pm}=\frac{1}{2}\left[r \pm \sqrt{q-4\left(\lambda_{2}-\lambda_{1}\right) f}\right] .
$$

We let $\boldsymbol{\kappa}=\left[\kappa_{+}, \kappa_{-}\right]^{\top}$. By Theorem 2.1, any unitarily invariant norm is a superposition of a symmetric gauge with the singular value map. Therefore, by symmetry of $\mathbf{Y}-\mathbf{N}$, it is true that

$$
\|\mathbf{Y}-\mathbf{N}\|=|\boldsymbol{\kappa}|
$$

for some symmetric gauge $|\cdot|$. Let $w: \mathbb{R}_{+}^{2} \rightarrow \mathbb{R}_{+}$be a function that coincides with the symmetric gauge in the nonnegative quadrant (thus, for the $p$-norm, $w(\mathbf{x})=\sqrt[p]{x_{1}^{p}+x_{2}^{p}}$ ). Assuming that $w$ is differentiable for $\mathbf{x} \neq \mathbf{0}$ in the positive quadrant, we denote its derivatives by

$$
w_{1}=\frac{\partial w}{\partial x_{1}}, \quad w_{2}=\frac{\partial w}{\partial x_{2}},
$$

and note that, being a norm, $|\cdot|$ is a weakly convex function. Thus, it is necessarily true that

$$
w_{1}(\mathbf{x}), w_{2}(\mathbf{x}), \frac{w_{1}(\mathbf{x})-w_{2}(\mathbf{x})}{x_{1}-x_{2}} \geq 0, \quad x_{1}, x_{2} \geq 0, x_{1}+x_{2}>0, x_{1} \neq x_{2} .
$$


(In the case when $|\cdot|$ is not differentiable in the open positive quadrant, we may approximate it arbitrarily closely by a differentiable symmetric gauge.) Note that we do not need to bother ourselves with the case $x_{1}=x_{2}$, since, by (3.7), away from equilibria, the eigenvalues $\kappa_{ \pm}$are distinct.

Because of the symmetry of $|\cdot|$, it is enough to consider the case $0<x_{1}<x_{2}$, when also $w_{1} \pm w_{2} \geq 0$. We have

$$
\frac{d}{d t}\|\mathbf{Y}-\mathbf{N}\|=\frac{d}{d t}|\boldsymbol{\kappa}|=w_{1} \frac{d\left|\kappa_{+}\right|}{d t}+w_{2} \frac{d\left|\kappa_{2}\right|}{d t}
$$

Therefore, if $\kappa_{+} \kappa_{2} \geq 0$, then

$$
\frac{d}{d t}\|\mathbf{Y}-\mathbf{N}\|=-\left(w_{1}-w_{2}\right) \frac{\left(\lambda_{2}-\lambda_{1}\right) f^{\prime}}{\sqrt{q-4\left(\lambda_{2}-\lambda_{1}\right) f}},
$$

while in the case $\kappa_{+} \kappa_{-}<0$, we obtain

$$
\frac{d}{d t}\|\mathbf{Y}-\mathbf{N}\|=-\left(w_{1}+w_{2}\right) \frac{\left(\lambda_{2}-\lambda_{1}\right) f^{\prime}}{\sqrt{q-4\left(\lambda_{2}-\lambda_{1}\right) f}} .
$$

Because of (3.4), it is true that $\left(\lambda_{2}-\lambda_{1}\right) f^{\prime} \geq 0$. Therefore, $d\|\mathbf{Y}-\mathbf{N}\| / d t \leq 0$, hence we obtain global optimality.

We turn our attention to isospectral optimality and distinguish, along the lines of the analysis in Section 2, between weak and strong optimality.

Let $|\cdot|$ be a symmetric gauge. Because $|\mathbf{x}|=\|\mathbf{x}\|$, it is not a loss of generality to assume again that $\mathbf{x} \in \mathbb{R}^{2}$ lies in the positive quadrant $\mathbb{R}_{+}^{2}$. We assume that it is differentiable for $x_{1}, x_{2}>0$ and, resurrecting the terminology of Section 2, let

$$
g_{k}(\mathbf{x})=\frac{\partial|\mathbf{x}|}{\partial x_{k}}, \quad k=1,2, \quad v(\mathbf{x})=\frac{g_{1}(\mathbf{x})-q_{2}(\mathbf{x})}{x_{1}-x_{2}} .
$$

THEOREM 3.2. Every unitarily invariant norm is weakly isospectrally optimal. If

$$
v(\mathbf{x})>0, \quad x_{1}, x_{2}>0, \quad x_{1} \neq x_{2},
$$

then the norm is strongly isospectrally optimal.

Proof. It follows from the convexity of the ball of radius $|\mathbf{x}|$ that $g_{1}, g_{2}, v \geq 0$. The key to isospectral optimality is the inequality (2.11). For $n=2$, though, we have

$$
\mu_{1}=\frac{U_{1,2}}{\eta_{1}-\eta_{2}}, \quad \mu_{2}=-\mu_{1}
$$

therefore,

$$
\boldsymbol{\mu}^{\top} \mathbf{g}=U_{1,2} \frac{g_{1}(|\boldsymbol{\eta}|)-g_{2}(|\boldsymbol{\eta}|)}{\eta_{1}-\eta_{2}}
$$

We distinguish between two cases. If $\eta_{1} \eta_{2}>0$, we may assume without loss of generality that $\eta_{1}, \eta_{2}>0$, (otherwise we multiply both $\mathbf{Y}_{0}$ and $\mathbf{N}$ by -1 in (1.1)). In that case, (3.17) 
implies that

$$
\boldsymbol{\mu}^{\top} \mathbf{g}=U_{1,2} v(\boldsymbol{\eta})
$$

and, $U_{1,2}$ being positive, $\boldsymbol{\mu}^{\top} \mathbf{g}>0$ if and only if $v(\boldsymbol{\eta})>0$. In the second case, $\max \left\{\eta_{k}\right\}>$ $0>\min \left\{\eta_{k}\right\}$, we may assume without loss of generality that $\eta_{1}>0>\eta_{2},\left|\eta_{1}\right| \geq\left|\eta_{2}\right|$ (otherwise, again, we change signs in (1.1)). Therefore,

$$
\boldsymbol{\mu}^{\top} \mathbf{g}=U_{1,2} \frac{\left|\eta_{1}\right|-\left|\eta_{2}\right|}{\left|\eta_{1}\right|+\left|\eta_{2}\right|} v(|\boldsymbol{\eta}|)
$$

and, again, $\boldsymbol{\mu}^{\top} \mathbf{g}>0$ is equivalent to $v(|\boldsymbol{\eta}|)>0$. Note that, by the triangle inequality and symmetry,

$$
\begin{aligned}
& \left|\left[\alpha x_{2}+(1-\alpha) x_{1}, \alpha x_{1}+(1-\alpha) x_{2}\right]^{\top}\right| \\
& \quad \leq \alpha\left|\left[x_{2}, x_{1}\right]^{\top}\right|+(1-\alpha)\left|\left[x_{1}, x_{2}\right]^{\top}\right|=|\mathbf{x}|
\end{aligned}
$$

for every $\alpha \in(0,1)$, therefore,

$$
\frac{1}{\alpha}\left[\left|\left[\alpha x_{2}+(1-\alpha) x_{1}, \alpha x_{1}+(1-\alpha) x_{2}\right]^{\top}\right|-\left|\left[x_{1}, x_{2}\right]^{\top}\right|\right] \geq 0
$$

Letting $\alpha \downarrow 0$, it follows that $v(\mathbf{x}) \geq 0$. We thus deduce from (2.11) that, while every unitarily invariant norm is weakly isospectrally optimal, it is strongly isospectrally optimal if and only if (3.15) holds.

To illustrate Theorem 3.2, we commence by considering $|\cdot|_{p}, 1 \leq p \leq \infty$, corresponding to Schatten $p$-norms. If $p \in(1, \infty)$, we obtain

$$
v(\mathbf{x})=|\mathbf{x}|_{p}^{1-p} \frac{x_{1}^{p-1}-x_{2}^{p-1}}{x_{1}-x_{2}}
$$

Predictably, $v(\mathbf{x})>0$ for $1<p<\infty$. For $p=1$, we obtain $v(\mathbf{x}) \equiv 0$ : again, hardly a surprise, considering that we already know from Theorem 2.2 that the Ky Fan norm is weakly isospectrally optimal. Finally, for $p=\infty$ it is easy to compute

$$
v(\mathbf{x})=\frac{1}{\left|x_{1}-x_{2}\right|}>0
$$

and again we obtain strong isospectral optimality. Note that $v$ blows up along the line $x_{1}=x_{2}$, but this, as we have already commented, does not interfere with our analysis. 


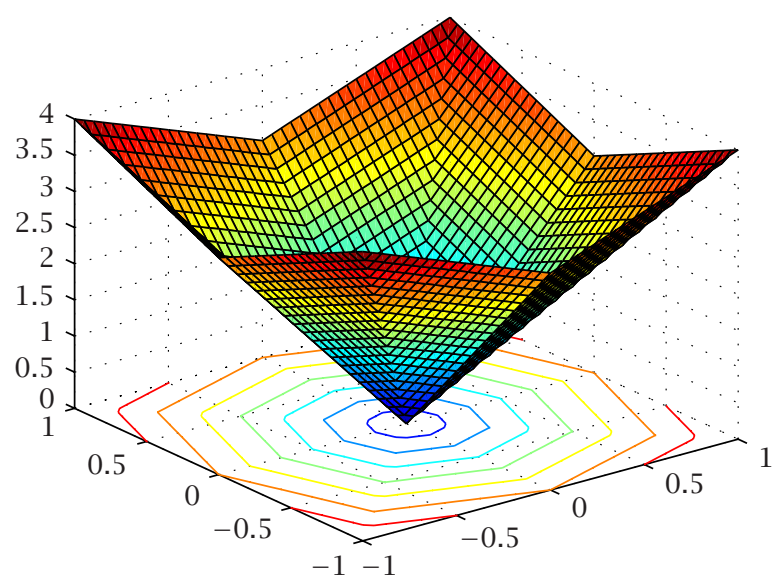

(a)

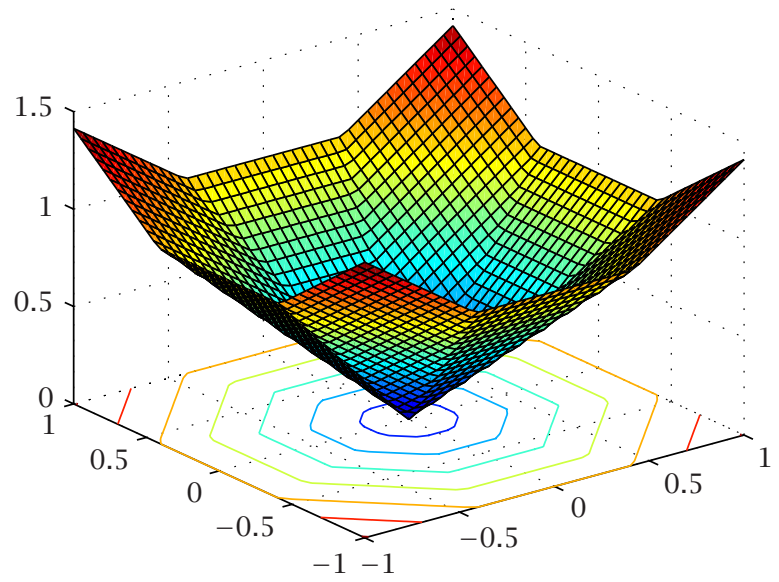

(b)

FIGURE 3.1. The norms (3.24) (a) and (3.25) (b).

We present three examples, illustrated in order in Figures 3.1 and 3.2, of more "exotic" symmetric gauges (in each case it is easy to prove that the underlying function is, in fact, a symmetric gauge), the first being

$$
|\mathbf{x}|=\left|x_{1}\right|+\left|x_{2}\right|+\left|x_{1}+x_{2}\right|+\left|x_{1}-x_{2}\right|, \quad \mathbf{x} \in \mathbb{R}^{2} .
$$

Therefore, $v(\mathbf{x})=2 /\left|x_{1}-x_{2}\right|$ and strong isospectral optimality follows from (3.15). ( $v$ does not exist for $x_{1}=x_{2}$, where the norm is not differentiable, but this is absolutely no hindrance.) The figures illustrate the norm as a function of $x_{1}$ and $x_{2}$. 


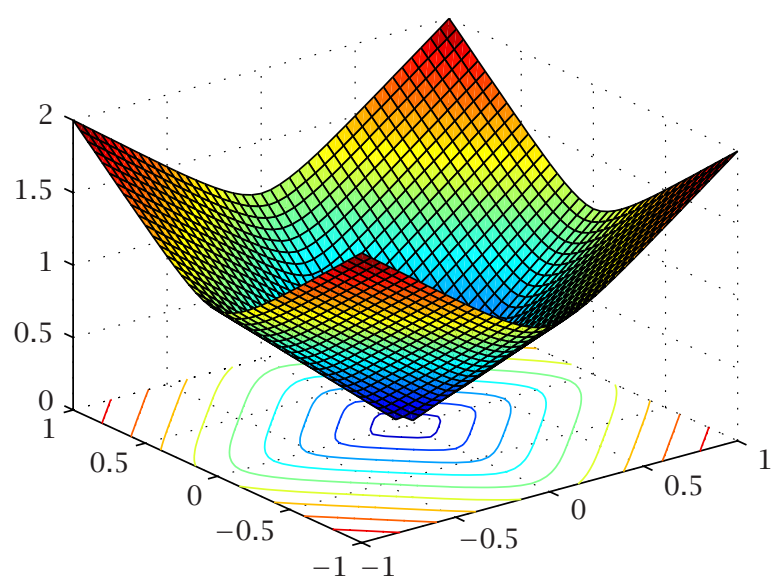

FIGURE 3.2. The norm (3.26).

To illustrate breakdown in strong optimality, our next example is based on the intuitive notion that $\min v(\mathbf{x})=0$ means that the norm is somehow "similar" to $|\cdot|_{1}$ near the line $x_{1}=x_{2}$. Thus, we let

$$
|\mathbf{x}|= \begin{cases}\left|x_{1}\right|, & \left|x_{2}\right| \leq(\sqrt{2}-1)\left|x_{1}\right| \\ \left|x_{2}\right|, & \left|x_{1}\right| \leq(\sqrt{2}-1)\left|x_{2}\right| \\ \frac{\sqrt{2}}{2}\left(\left|x_{1}\right|+\left|x_{2}\right|\right), & || x_{1}|-| x_{2}|| \leq(2-\sqrt{2}) \max \left\{\left|x_{1}\right|,\left|x_{2}\right|\right\}\end{cases}
$$

Therefore, $v \equiv 0$ in a wedge surrounding $x_{1}=x_{2}$ and strong isospectral optimality is lost.

Our final example is a symmetric gauge that produces $v=0$ along the line $x_{1}=x_{2}$, but nowhere else,

$$
|\mathbf{x}|=\left|x_{1}\right|+\left|x_{2}\right|+\frac{1}{7} \frac{\left(\left|x_{1}\right|-\left|x_{2}\right|\right)^{4}}{\left(\left|x_{1}\right|+\left|x_{2}\right|\right)^{3}}
$$

The constant $1 / 7$ ensures continuity and it is easy to prove that (3.26) is a symmetric gauge: the only nontrivial item is the satisfaction of the triangle inequality, which follows at once from the observation that the unit ball of (3.26) is convex. We have

$$
v(\mathbf{x})=\frac{8}{7} \frac{\left(\left|x_{1}\right|-\left|x_{2}\right|\right)^{2}}{\left(\left|x_{1}\right|+\left|x_{2}\right|\right)^{3}}>0, \quad x_{1}, x_{2}>0, x_{1} \neq x_{2},
$$

hence we obtain strong isospectral optimality. This serves to emphasize the important point that strong isospectral optimality depends on the behavior of the symmetric gauge near, but not on, the line $x_{1}=x_{2}$. 
4. Further remarks on general $n \geq 2$. The results of Sections 2 and 3 , as well as a large volume of numerical computations, are highly suggestive of a conjecture that the attractive fixed point is globally optimal with respect to every unitarily invariant norm.

Depending on the specific norm, numerical experiments highlight two types of behavior: if the norm in question is strictly isospectrally optimal, $\|\mathbf{Y}(t)-\mathbf{N}\|$ decreases strictly monotonically until $t$ reaches $\infty$. However, if we have only weak isospectral optimality, it is perfectly possible for $\|\mathbf{Y}(t)-\mathbf{N}\|$ to decrease strictly monotonically for a while and then hit a "plateau": from that moment, $\|\mathbf{Y}(t)-\mathbf{N}\|$ remains fixed at its optimal value, although we might be far away from the fixed point.

It is useful and highly illuminating to consider symmetric matrices along an isospectral orbit by examining their Horn-Schur polytope. Given a vector $\boldsymbol{\kappa} \in \mathbb{R}^{n}$, the Horn-Schur polytope $\mathscr{H}$ is the closed convex hull of the $n$ ! permutations of $\boldsymbol{\kappa}$ [13]. The following features of the Horn-Schur polytope underlie its relevance to the analysis of DBF.

(i) Given any $\mathbf{A} \in \operatorname{Sym}(n)$ with $\sigma(\mathbf{A})=\boldsymbol{\kappa}$, necessarily diag $\mathbf{A} \in \mathcal{H}$. In particular, if $\mathbf{Y}(t)$ is the solution of (1.1), then its diagonal forms a trajectory in the Horn-Schur polytope generated by the eigenvalues of $\mathbf{Y}_{0}$.

(ii) Given any $\mathbf{x} \in \mathscr{H}$, there exists $\mathbf{A} \in \operatorname{Sym}(n)$ such that $\sigma(\mathbf{A})$ is given by the vertices of $\mathscr{H}$ and $\operatorname{diag} \mathbf{A}=\mathbf{x}$. (Such a matrix A need not be unique.) Therefore, having the freedom to choose $\mathbf{Y}_{0}$ in (1.1), we can commence the trajectory diag $\mathbf{Y}(t)$ at any point in the polytope.

(iii) The diagonal of $\hat{\mathbf{Y}}$ is a vertex of $\mathscr{H}$, since the attractive fixed point is a diagonal matrix. Therefore, one privileged vertex of $\mathscr{H}$ attracts all orbits commencing in the interior of the polytope.

We focus on the Ky Fan norm which, according to Theorem 2.2, is weakly isospectrally optimal. Denoting $\boldsymbol{v}(t)=\sigma(\mathbf{Y}(t)-\mathbf{N})$, we observe at once that, as long as all the elements of $v$ are of the same sign, that is, if either $\min v_{k} \geq 0$ or $\max v_{k} \leq 0$, then

$$
\|\mathbf{Y}(t)-\mathbf{N}\|_{1}=|\boldsymbol{v}|_{1}=\mathbf{1}^{\top}|\boldsymbol{v}|=\left|\mathbf{1}^{\top} \boldsymbol{v}\right|=|\operatorname{tr}(\mathbf{Y}(t)-\mathbf{N})| \equiv\left|\mathbf{1}^{\top}(\mathbf{d}-\boldsymbol{\lambda})\right|
$$

Hence, in that instance the norm does not vary as $t$ increases. By the same token, if the signs of $\boldsymbol{v}$ vary, it follows at once that $\|\mathbf{Y}(t)-\mathbf{N}\|_{1}>\left|\mathbf{1}^{\top}(\mathbf{d}-\boldsymbol{\lambda})\right|$. It thus makes sense to partition the polytope $\mathscr{H}$, whose vertices are determined by the eigenvalues of $\mathbf{Y}_{0}$, into the following three subsets.

(1) All points $\mathbf{x} \in \mathcal{H}$ such that the eigenvalues of $\mathbf{Y}-\mathbf{N}$ for every $\mathbf{Y} \in \operatorname{Sym}(n), \operatorname{diag} \mathbf{Y}=$ $\mathbf{X}, \sigma(\mathbf{Y})=\sigma\left(\mathbf{Y}_{0}\right)$, are of the same sign (denoted in Figure 4.1 by black).

(2) All points $\mathbf{x} \in \mathcal{H}$ such that the eigenvalues of $\mathbf{Y}-\mathbf{N}$ for every $\mathbf{Y} \in \operatorname{Sym}(n), \operatorname{diag} \mathbf{Y}=$ $\mathbf{x}, \sigma(\mathbf{Y})=\sigma\left(\mathbf{Y}_{0}\right)$, change sign (denoted in Figure 4.1 by light grey).

(3) All points $\mathbf{x} \in \mathscr{H}$ such that there exist $\mathbf{Y}_{1}, \mathbf{Y}_{2} \in \operatorname{Sym}(n)$ with $\operatorname{diag} \mathbf{Y}_{1}=\operatorname{diag} \mathbf{Y}_{2}=\mathbf{x}$, $\sigma\left(\mathbf{Y}_{1}\right)=\sigma\left(\mathbf{Y}_{2}\right)=\sigma\left(\mathbf{Y}_{0}\right)$, the eigenvalues of $\mathbf{Y}_{1}-\mathbf{N}$ are all of the same sign and the eigenvalues of $\mathbf{Y}_{2}-\mathbf{N}$ change sign (denoted in Figure 4.1 by dark grey).

Since $\mathbf{1}^{\top} \mathbf{x}$ is constant for all $\mathbf{x} \in \mathscr{H}$, the Horn-Schur polytope is an $(n-1)$-dimensional object. In particular, given $n=3$, $\mathscr{H}$ "lives" on a hyperplane that can be mapped on the plane $\mathbb{R}^{2}$ : a rotation of the hyperplane is probably much more instructive than projection and it has been employed in Figure 4.1. 


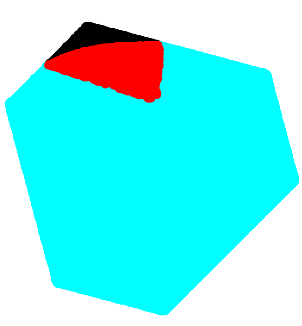

(a)

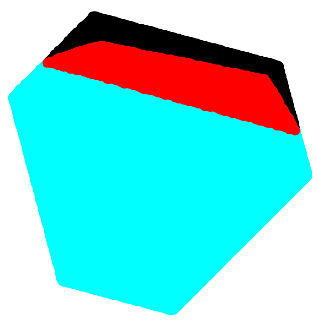

(b)

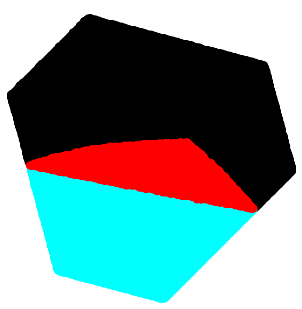

(c)

FIgURE 4.1. The partition of the Horn-Schur polytope for $\mathbf{d}=[0,3 / 4,2]^{\top}$ and for (a) $\boldsymbol{\lambda}=[1 / 2,3 / 2,3]^{\top}$, (b) $\boldsymbol{\lambda}=[1 / 2,3]^{\top}$, and (c) $\boldsymbol{\lambda}=[3 / 2,5 / 2,3]^{\top}$.

There are three possibilities: sometimes the entire polytope is "black," sometimes it is "light grey", and sometimes it includes all three colours. By inspection, everything depends on the relative sizes of $\boldsymbol{\lambda}$ and $\mathbf{d}$ (where, without loss of generality, we assume that each vector has monotonically increasing entries). We denote $d_{k}$ by "•" and $\lambda_{k}$ by "॰," therefore, for example, $\bullet \circ \circ \bullet$ corresponds to the configuration $d_{1}<\lambda_{1}<\lambda_{2}<d_{2}$. In $\mathbb{R}^{3}$, out of all possible configurations, just two, $\circ \circ \circ \bullet \bullet$ and $\bullet \bullet \bullet \circ \circ$, give a polytope which is entirely "black" (hence, the Ky Fan norm is constant along all trajectories). Eight further configurations,

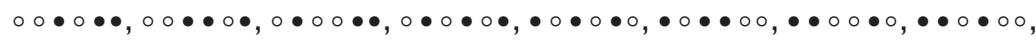

result in a "mixed" polytope, while all remaining choices produce a "light grey" polytope, where the Ky Fan norm strictly decreases along every trajectory. Figure 4.1 displays three instances of "mixed" polytopes.

The "exotic norms" (3.25) and (3.26) can be generalized to arbitrary $n \geq 2$. To generalize (3.25), we meld $|\cdot|_{1}$ and $|\cdot|_{\infty}$ : the first near the line $x_{1}=x_{2}=\cdots=x_{n}$ and its symmetric "replicas" in other orthants, the other elsewhere, while ensuring continuity and convexity. As long as all the eigenvalues of $\mathbf{Y}-\mathbf{N}$ lie in the positive orthant, within the $n$-dimensional wedge where the norm behaves like $|\cdot|_{1}$, we experience weak isospectral optimality.

We generalize (3.26) by setting

$$
|\mathbf{x}|=|\mathbf{x}|_{1}+\frac{1}{7(n-1)} \sum_{k=1}^{n-1} \sum_{l=k+1}^{n} \frac{\left(\left|x_{k}\right|-\left|x_{l}\right|\right)^{4}}{\left(\left|x_{k}\right|+\left|x_{l}\right|\right)^{3}} .
$$

This is indeed a symmetric gauge. The only difficulty is in proving that the unit ball with respect to the function above is locally convex along the "joins" between orthants. Yet, considering tangents therein, it is possible to prove convexity (which implies the triangle inequality) also when $1 / 7$ is replaced by a smaller positive number. Although we have not proved that this norm is strongly isospectrally optimal, numerical calculations 
indicate that this is probably the case. Thus, it is not enough for a norm to "resemble" $|\cdot|_{1}$ just along the line $x_{1}=x_{2}=\cdots=x_{n}$ for isospectral optimality to be weak.

5. Conclusions and relationship to the Horn conjectures. We conclude this paper by making some comparisons to recent work on the Horn conjectures. In particular, we show that these results, while related to the work here, do not imply the optimality results above.

A key role is this paper is played by the matrix $-\mathbf{N}+\mathbf{Y}$. There has been a great deal of interest recently in the relationship of the eigenvalues of the sum of two symmetric (or Hermitian) matrices to the eigenvalues of the summands. A nice survey of the history and current state of the art of this problem may be found in [11], while the relationship of this problem to combinatorial objects known as "honeycombs" is discussed in [16]. A general list of inequalities between triples $(\alpha, \beta, \gamma)$ of $n$-vectors such that they occur as the eigenvalues of symmetric or Hermitian matrices $(A, B, C)$ with $A+B=C$ was conjectured in [12] and recently proved in a series of papers, as described in [11].

The general case may be clearly reduced to our case of a diagonal matrix summed with a general symmetric matrix, viewed as the conjugation of a diagonal matrix by orthogonal matrices.

We consider the Horn conjectures as applied then to the sum $-\mathbf{N}+\mathbf{Q Y} \mathbf{Y}_{0} \mathbf{Q}^{\top}$. We do not consider the full set of conjectures but note some special (earlier) necessary conditions that are of interest.

Firstly, from [26] one has (using our earlier notation)

$$
v_{i+j-1} \leq-\lambda_{i}+d_{j} \text { for } i+j-1 \leq n
$$

Fan found the necessary conditions

$$
\sum_{i=1}^{r} v_{i} \leq-\sum_{i=1}^{r} \lambda_{i}+\sum_{i=1}^{r} d_{i} \quad \text { for any } r<n
$$

(see [10]) and Lidskiǐ showed that, viewing the $n$-tuples of eigenvalues as vectors in $\mathbb{R}^{n}$, $v$ must lie in the convex hull of $-\lambda+d_{\sigma}$, where $\sigma$ denotes all permutations over the symmetric group [20]. Wielandt in turn showed that this latter condition was equivalent to the set of inequalities

$$
\sum_{i \in I} v_{i} \leq-\sum_{i \in I} \lambda_{i}+\sum_{i=1}^{r} d_{i}
$$

for every subset $I$ of $\{1, \ldots, n\}$ of cardinality $r$ for all $r<n$ [27].

One observes then that the spectrum of $-\mathbf{N}+\mathbf{Q Y} \mathbf{Y}_{0} \mathbf{Q}^{\top}$ lies in a polytope which is shifted from the Schur-Horn polytope by the vector $\boldsymbol{\lambda}$. That the eigenvalues occur in the convex hull of this shifted polytope rather than diagonals is due to the fact that this polytope arises as the image of a different momentum map. In the Schur-Horn case, the relevant map arises from a torus action on an adjoint orbit, while the shifted polytope arises from the action of the unitary group on a cross product of orbits. 
We note that the inequalities above and indeed the Horn inequalities in general do not imply the inequalities $v_{i} \leq-\lambda_{i}+d_{i}$ except for $n=1$ and thus do not imply the optimality results discussed above. Further, it is interesting that the remaining optimality results used in this paper rest on nonlinear functions of the eigenvalues of the relevant matrices and are thus also beyond the scope of both the Horn inequalities and the Schur-Horn inequalities. The latter inequalities are in fact a special case of the former (see [19]).

ACKNOWLEDGMENTS. We would like to thank Bill Fulton and Sergey Fomin for useful discussions. The work of Anthony M. Bloch was supported in part by the National Science Foundation.

\section{REFERENCES}

[1] A. M. Bloch, Steepest descent, linear programming, and Hamiltonian flows, Mathematical Developments Arising from Linear Programming (Brunswick, Me, 1988), Contemp. Math., vol. 114, American Mathematical Society, Rhode Island, 1990, pp. 77-88.

[2] A. M. Bloch, R. W. Brockett, and T. S. Ratiu, A new formulation of the generalized Toda lattice equations and their fixed point analysis via the momentum map, Bull. Amer. Math. Soc. (N.S.) 23 (1990), no. 2, 477-485.

[3] Completely integrable gradient flows, Comm. Math. Phys. 147 (1992), no. 1, 57-74.

[4] A. M. Bloch, H. Flaschka, and T. S. Ratiu, A convexity theorem for isospectral manifolds of Jacobi matrices in a compact Lie algebra, Duke Math. J. 61 (1990), no. 1, 41-65.

[5] R. W. Brockett, Dynamical systems that sort lists, diagonalize matrices, and solve linear programming problems, Linear Algebra Appl. 146 (1991), 79-91.

[6] M. P. Calvo, A. Iserles, and A. Zanna, Numerical solution of isospectral flows, Math. Comp. 66 (1997), no. 220, 1461-1486.

[7] M. T. Chu and K. R. Driessel, Constructing symmetric nonnegative matrices with prescribed eigenvalues by differential equations, SIAM J. Math. Anal. 22 (1991), no. 5, 13721387.

[8] P. Deift, T. Nanda, and C. Tomei, Ordinary differential equations and the symmetric eigenvalue problem, SIAM J. Numer. Anal. 20 (1983), no. 1, 1-22.

[9] P. A. Deift, S. Rivera, C. Tomei, and D. S. Watkins, A monotonicity property for Toda-type flows, SIAM J. Matrix Anal. Appl. 12 (1991), no. 3, 463-468.

[10] K. Fan, On a theorem of Weyl concerning eigenvalues of linear transformations. I, Proc. Nat. Acad. Sci. U.S.A. 35 (1949), 652-655.

[11] W. Fulton, Eigenvalues, invariant factors, highest weights, and Schubert calculus, Bull. Amer. Math. Soc. (N.S.) 37 (2000), no. 3, 209-249.

[12] A. Horn, Eigenvalues of sums of Hermitian matrices, Pacific J. Math. 12 (1962), 225-241.

[13] R. A. Horn and C. R. Johnson, Topics in Matrix Analysis, Cambridge University Press, Cambridge, 1991.

[14] A. Iserles, On the discretization of double-bracket flows, Found. Comput. Math. 2 (2002), no. 3, 305-329.

[15] A. Iserles, H. Z. Munthe-Kaas, S. P. Nørsett, and A. Zanna, Lie-group methods, Acta Numerica, 2000, Acta Numer., vol. 9, Cambridge University Press, Cambridge, 2000, pp. 215-365.

[16] A. Knutson and T. Tao, Honeycombs and sums of Hermitian matrices, Notices Amer. Math. Soc. 48 (2001), no. 2, 175-186.

[17] J. Lagarias, Monotonicity properties of the Toda flow, the QR-flow, and subspace iteration, SIAM J. Matrix Anal. Appl. 12 (1991), no. 3, 449-462. 
[18] A. S. Lewis and M. L. Overton, Eigenvalue optimization, Acta Numerica, 1996, Acta Numer., vol. 5, Cambridge University Press, Cambridge, 1996, pp. 149-190.

[19] C.-K. Li and Y.-T. Poon, Principal submatrices of a Hermitian matrix, Linear Multilinear Algebra 51 (2003), no. 2, 199-208.

[20] V. B. Lidskiĭ, On the characteristic numbers of the sum and product of symmetric matrices, Dokl. Akad. Nauk SSSR (N.S.) 75 (1950), 769-772.

[21] J. Moser, Finitely many mass points on the line under the influence of an exponential potential - an integrable system, Dynamical Systems, Theory and Applications (Rencontres, BattelleRes. Inst., Seattle, Wash., 1974), Lecture Notes in Phys., vol. 38, Springer-Verlag, Berlin, 1975, pp. 467-497.

[22] W. W. Symes, Hamiltonian group actions and integrable systems, Phys. D 1 (1980), no. 4, 339-374.

[23] _ The QR algorithm and scattering for the finite nonperiodic Toda lattice, Phys. D 4 (1982), no. 2, 275-280.

[24] J. von Neumann, Some matrix inequalities and metrization of matric space, Tomsk University Rev. 1 (1937), 286-300.

[25] - Some matrix inequalities and metrization of matric space, Collected Works. Volume 4: Continuous Geometry and Other Topics (A. H. Taub, ed.), Pergamon, Oxford, 1962, pp. 205-218.

[26] H. Weyl, Das asymptotische Verteilungesetz der Eigenwerte lineare partieller Differentialgleichungen, Math. Ann. 71 (1912), 441-479 (German).

[27] H. Wielandt, An extremum property of sums of eigenvalues, Proc. Amer. Math. Soc. 6 (1955), 106-110.

[28] J. H. Wilkinson, The Algebraic Eigenvalue Problem, Clarendon Press, Oxford, 1965.

Anthony M. Bloch: Department of Mathematics, University of Michigan, Ann Arbor, MI 48109, USA

E-mail address: ab7och@umich.edu

Arieh Iserles: Department of Applied Mathematics and Theoretical Physics, Centre for Mathematical Sciences, University of Cambridge, Wilberforce Road, Cambridge CB3 OWA, UK

E-mail address: a.i serles@damtp.cam.ac.uk 


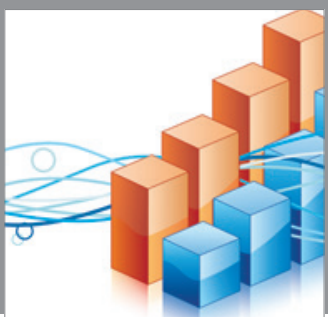

Advances in

Operations Research

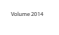

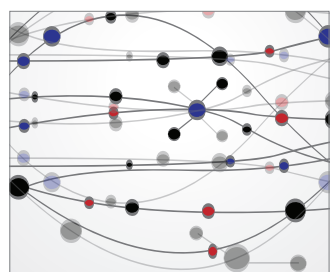

\section{The Scientific} World Journal
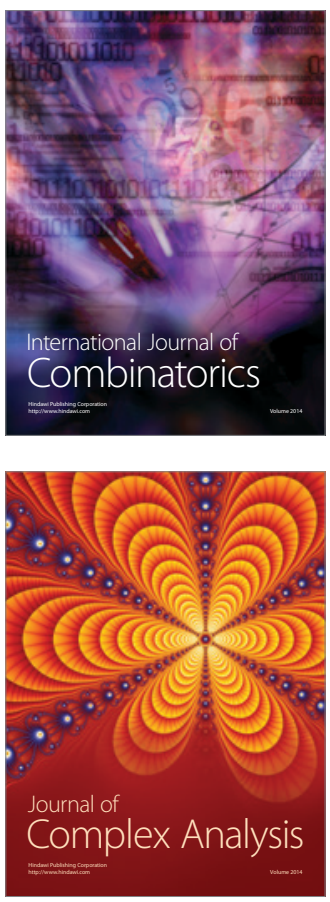

International Journal of

Mathematics and

Mathematical

Sciences
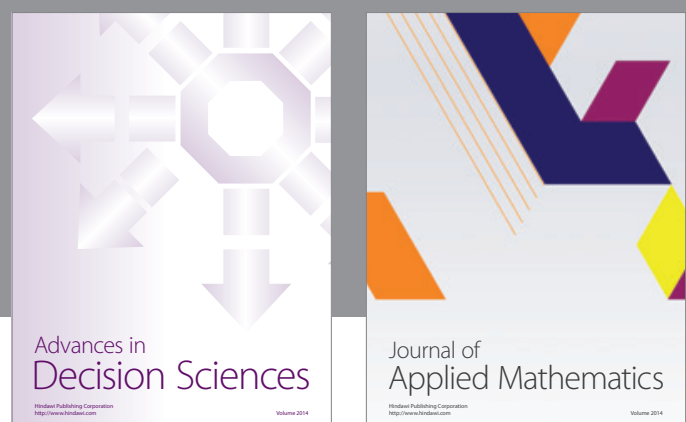

Journal of

Applied Mathematics
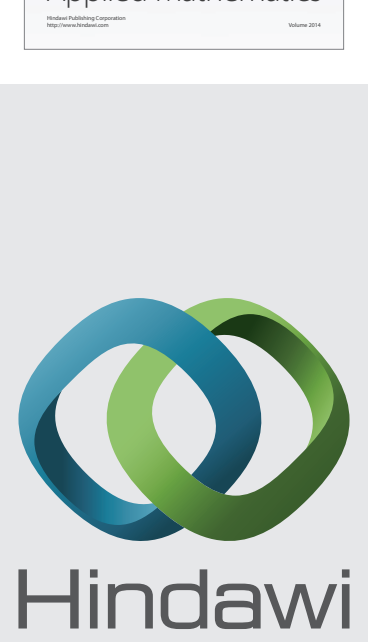

Submit your manuscripts at http://www.hindawi.com
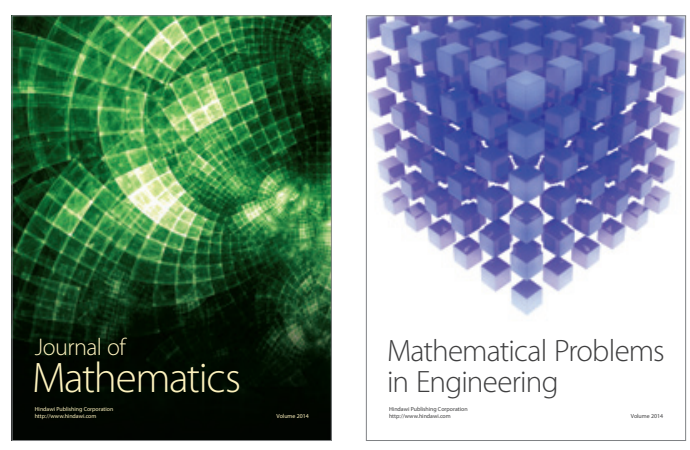

Mathematical Problems in Engineering
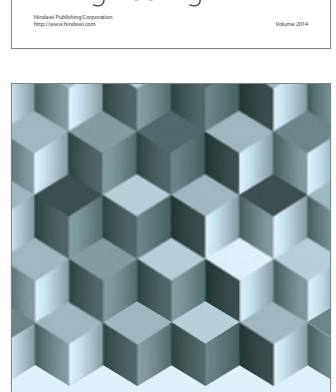

Journal of

Function Spaces
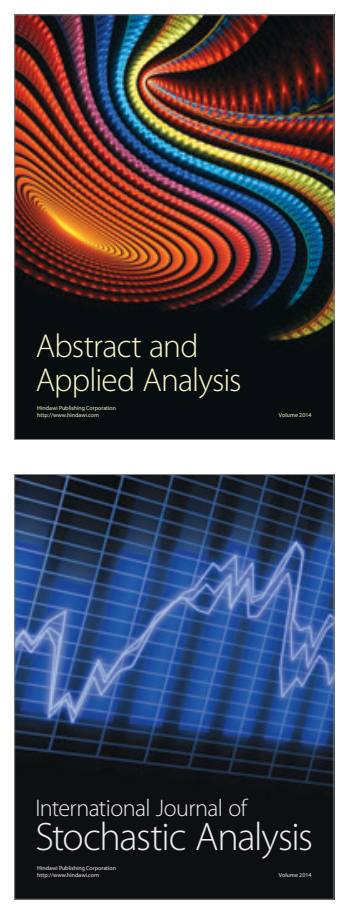

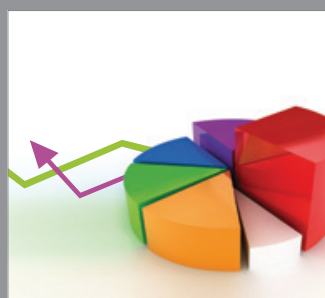

ournal of

Probability and Statistics

Promensencen
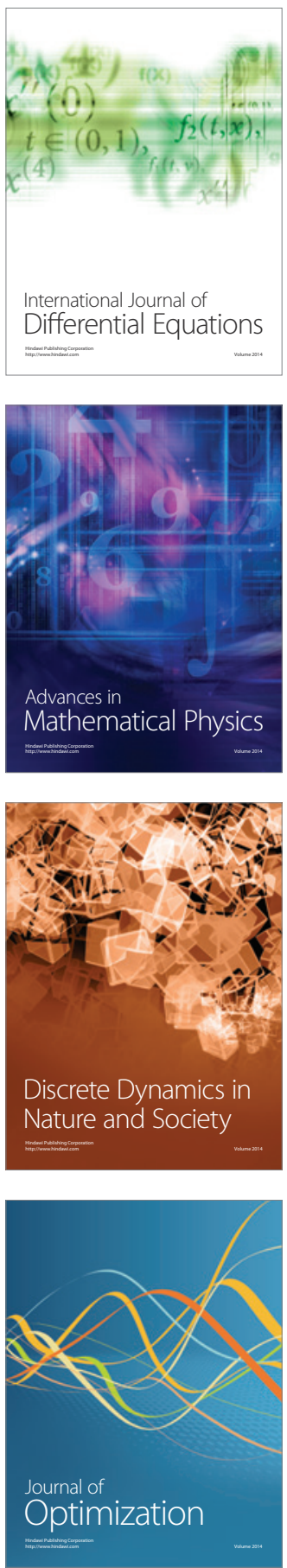